\title{
Reduced electrodermal response to errors predicts poor sustained attention performance in Attention Deficit Hyperactivity Disorder
}

Redmond G. O’Connell ${ }^{1}$, Mark A. Bellgrove ${ }^{1,2}$, Paul M. Dockree ${ }^{1}$ \& Ian H. Robertson ${ }^{1}$

${ }^{1}$ Department of Psychology and Trinity College Institute of Neuroscience, Trinity College, Dublin 2, Ireland.

${ }^{2}$ Department of Psychiatry, Trinity College, Dublin 2, Ireland

Correspondence: Redmond O’Connell, Department of Psychology, Trinity College, Dublin 2, Ireland Tel: + 3531608 3912. Email: oconnelr@tcd.ie

Running Head: EDA and sustained attention in ADHD

\section{Acknowledgments}

The work reported herein was supported by the Government of Ireland Research

Scholarship in the Humanities and Social Sciences to R. G. O’Connell and is administered by the Irish Research Council for the Humanities and Social Sciences.

M.A. Bellgrove is supported by the Irish Health Research Board. The authors would also like to acknowledge the support of the Higher Education Authority of Ireland and all the children and families who participated in this study. 


\section{Abstract}

Research into ADHD has indicated abnormalities in electrodermal system activity (EDA) and separately, deficits in sustained attention. Here we asked for the first time whether reduced EDA in ADHD was consequential for the attention problems associated with this disorder. On a sustained attention task ADHD participants had higher overall error rates and exhibited a marked decrease in skin conductance responses (SCRs) to errors, relative to control children. Further, sustained attention errors were predicted by SCR amplitudes. Evidence of post-error slowing in both groups suggested comparable error awareness. It is proposed that attenuation of the normal autonomic response to errors may impair ADHD participants' psychological response to error significance and result in decreased behavioural correction and poorer sustained attention.

Keywords: Electrodermal activity, sustained attention, post-error correction, ADHD 


\section{Introduction}

ADHD is a highly prevalent child developmental disorder with core behavioural symptoms of age-inappropriate inattention, impulsivity and hyperactivity [1]. Though functional and structural imaging studies indicate fronto-striatal abnormalities (predominantly right lateralised) [2,3], the cognitive and neurological correlates of ADHD have not yet been firmly established and diagnosis is still based on exclusively behavioural criteria. In particular the attention problems associated with ADHD have been poorly characterised and are relatively underrepresented in the literature. Two consistent findings in ADHD research have been poor sustained attention [4-6] and attenuated electrodermal activity (EDA) [7-9]. The importance of EDA as a "somatic marker" for the consequences of different behaviours [10] has been well established in decision-making tasks however it is not clear how somatic markers are related to other executive functions such as, for example, sustained attention [11]. In the present study we investigate whether the attenuated task-related EDA of children with ADHD might contribute to their poor performance on sustained attention tasks.

Sustained attention refers to the endogenous maintenance of an alert state in the absence of exogenous input, and is achieved by a predominantly right-hemispheric neural network [12]. While the findings of studies using the continuous performance task (CPT) paradigm have been largely inconsistent and have been interpreted in different ways $[13,14]$ a sustained attention impairment has been reliably demonstrated in ADHD in studies that have employed the Sustained Attention to Response Test (SART) [6, Bellgrove et al, under review] or standardised tests of sustained attention [4,5]. Unlike traditional CPTs where participants monitor strings of non-target stimuli in order to respond to a rare target, the SART requires participants to respond to non-target stimuli while withholding their response on the appearance of the no-go target. The simplicity of the SART tends to encourage a routine response set, placing heavy demands on the individual's ability to 
endogenously maintain the overall goal of withholding to the no-go target during the intertarget intervals. This is particularly apparent in the fixed version SART where targets are presented within a completely predictable sequence of stimuli. Thus we argue that the SART is a more sensitive measure of momentary lapses of attention than conventional CPTs [15]. Further we have demonstrated the sensitivity of the SART for indexing selfreports of everyday attentional failures [15] and recently showed that the endogenous maintenance of attention on the SART activates the aforementioned right hemisphere alertness network [16].

EDA is controlled by sympathetic innervation of the autonomic nervous system and has been widely used in psychophysiology as an index of the psychological processing of stimulus properties. Importantly with respect to ADHD, human lesion studies [e.g. 17, 18] have revealed the role of right frontal brain areas in the central control of EDA. Zahn, Grafman and Tranel [18] found that rather than directly affecting EDA however, right frontal damage produces a selective impairment of the psychological processing of significant stimuli. A number of studies have examined EDA in ADHD and while no consistent differences in baseline EDA have been found [8], children with ADHD do consistently exhibit reduced electrodermal responsiveness to task-related stimuli [7-9] suggesting that ADHD, like frontal pathology, may also involve a selective EDA deficit. No studies, however, have investigated how attenuated autonomic system activity might actually relate to the cognitive deficits implicated in ADHD.

According to the 'somatic marker hypothesis' [10] bodily states of arousal provide feedback that forms an integral part of behaviour selection. Based on this theory we hypothesise that a reduced electrodermal response to making an error, the most psychologically significant event during SART performance, will reduce the likelihood of ADHD participants engaging in post-error correction of performance strategy and thus contribute to an increased rate of sustained attention failures. 


\section{Methods}

Participants

13 male and 2 female (1 left handed) ADHD participants were recruited from an existing participant panel used for our ongoing research [19]. The patient group had a mean age of $11.4(\mathrm{SD}=1.7)$ and a mean IQ, as assessed by the WISC-III, of $97.7(\mathrm{SD}=11.6)$. Each child received a diagnosis following a clinical assessment which included the Child and Adolescent Psychiatric Assessment (CAPA) [20], the Child ADHD Teacher telephone interview [21] and the revised Connors Parents Rating Scale - Long Version (CPRS-R:L) [22] administered by a trained interviewer. Diagnoses were made according to DSM-IV, DSM-III-R and ICD-10 criteria and exclusion criteria were any known neurological condition, psychosis or IQ less than 70. The presence of learning disability was assessed using the Reading and Spelling sub-tests of the Wide Range Achievement Test (WRAT-3) [23]. In this study 8 of the ADHD participants had a diagnosis of ADHD Combined type (ADHD/C), 3 had a diagnosis of ADHD Hyperactive type (ADHD/H) and 4 had a diagnosis of ADHD Inattentive type (ADHD/I). 11 members of the ADHD group also met criteria for other disorders including Oppositional Defiant Disorder and Conduct Disorder. Patients were withdrawn from stimulant medication 24 hours prior to testing.

15 right-handed control children (1 female) with a mean age of $11.2(\mathrm{SD}=1.5)$ and an average IQ of $112.1(\mathrm{SD}=14.8)$ were recruited from primary schools in the Dublin area. Control children were included if they scored less than 20 on the Conners' Parent Symptom Questionnaire [24], had no personal or familial history of ADHD or ADD and no personal history of neurological or psychiatric illness. Parental consent was obtained for all children in accordance with the ethical guidelines of the Department of Psychology, Trinity College Dublin and St James’ Hospital, Dublin, Ireland. 


\section{Materials and Methods}

Sustained Attention Task

The sustained attention task used in this investigation was a variant of the Sustained Attention to Response Test (SART) [7] that includes a dual-task element [25]. Previous work with this version of the SART has established its relationship to the original SART and its sensitivity to index transient lapses of attention [25].

Participants were presented with a series of digits in a fixed sequence from 1 to 9 and were required to press the left mouse button, in time with a response cue, after each digit (go-trials), except when the digit 3 was presented (no-go trial). The digits, masks and response cue were presented centrally in white on a computer monitor against a black background. The response cue, an emboldened cross, was designed to reduce within and between participant variability and minimises the potential for a speed/accuracy trade off.

This version of the SART included a secondary task requirement whereby participants responded to the occasional appearance of a grey-coloured digit by pressing the right mouse button. This secondary task was designed to increase the demand on attentional resources and thus reduce the likelihood of ceiling effects [25]. The presentation of grey-coloured digits was restricted to numbers 5 through 9 in order to avoid any interference with performance in the period before and immediately after the presentation of a target. To avoid confusion participants were told that there would be no grey 3s.

A full SART block consisted of 225 stimuli in total of which 179 were go-trials (requiring a left button press), 25 were no-go trials (requiring the withholding of responding on the 3 ) and 17 were grey trials (requiring a right button press) resulting in a total block duration of approximately 6 minutes. Each participant completed four SART blocks, two with 8 randomly presented auditory tones $(659 \mathrm{~Hz}, 30 \mathrm{~ms}$ duration, 62dB intensity) as a cue to concentrate on the task, and two without. Cues were presented between the numbers 5 and 9 inclusive, again to avoid any competition with the withhold 
response to the number 3 . The manipulation of alerting cues was included as part of a concurrent study examining their effect on sustained attention in ADHD. Statistical analyses revealed no effect of cues on any of the dependent variables between or within groups and therefore in the present study, results were analysed by collapsing across the alerting conditions. An analysis of SCRs to alerts is included below as a means of further verifying the specificity of EDA differences.

SART testing was preceded by a practice block that included 27 go-trials, 3 no-go trials and 6 grey trials. To reduce the influence of non-attentional processes, such as working memory, participants had to achieve 100\% accuracy, in terms of grey digit detection, on the practice block before they could proceed to test. A rest period of approximately 5 minutes was allowed between each block.

\section{Electrodermal Activity}

EDA measurements were taken from all participants during SART testing with a 5 channel BIOPAC MP30B unit, calibrated to record skin conductance level (SCL) and skin conductance responses (SCRs) in microsiemens ( $\mu \mathrm{S})$. Two Ag/AgCl BIOPAC electrodes, with contact areas of approximately $6 \mathrm{~mm}$, were filled with SIGNA electrode gell and secured with a velcro strap to the volar surface of the distal phalanges of the index and middle fingers of the participant's non-dominant hand. After a five-minute rest period to ensure skin hydration by the gel, the BIOPAC software was calibrated to the participants' own electrodermal parameters before SART testing began.

EDA data was analysed using BIOPAC Student Lab Pro software according to previously established criteria $[17,18]$. A rise in skin conductance level (SCL) was considered to be a response (SCR) if its onset was between one and five seconds after a particular event (presentation of no-go stimulus or alert). SCRs were measured by subtracting the SCL at stimulus onset from the peak SCL within the latency period. The criterion for the smallest acceptable SCR was set at $0.02 \mu \mathrm{S}$. Any response below this 
threshold was recorded as 0 . The amplitude of the largest SCR within this latency period was measured. 


\section{Results}

The patient and control groups were successfully matched for gender $\left[x^{2}=0.37\right.$, $\mathrm{df}=1, \mathrm{p}=0.54]$, handedness $\left[x^{2}=1.03, \mathrm{df}=1, \mathrm{p}=0.3\right]$, age $[t(28)=-0.015, \mathrm{p}=0.99]$, and WRAT composite score intervals $\left[x^{2}=9.53, \mathrm{df}=6, \mathrm{p}=0.146\right]^{1}$. The groups were not matched for IQ $[t(28) .=2.9, \mathrm{p}=0.006]$, IQ did not however correlate with any of the dependent measures and primary analyses for the behavioural measures included IQ as a covariate.

Table 1 displays the means, standard deviations and significance levels for between group differences on each of the behavioural and EDA measures. Relative to control participants, children with ADHD made more errors of commission (pressing on 3) and of omission (failing to press on a go-target) and exhibited greater GoRT (i.e. reaction times to go-digits) variability during SART performance indicating impaired sustained attention.

The two groups did not differ in their SCRs to successful withholds $[F(1,28)=0.142, \mathrm{p}=0.709]$ or to the alerting cues $[\mathrm{t}(1,28)=0.786, \mathrm{p}=0.449]$. A significant difference was found for SCRs to commission errors $[F(1,28)=3.95, \mathrm{p}=0.05]$ as a function of group. A Repeated-measures ANOVA with Response (mean SCR amplitude to correct withholds vs. mean SCR amplitude to commission errors) as the within-subjects factor and Group as the between subjects factor was carried out and a significant main effect of Response $[\mathrm{F}(1,28)=15.5, \mathrm{p}=0.001]$ and a Response by Group interaction $[\mathrm{F}=4.72, \mathrm{p}=0.038]$ were found.

\footnotetext{
${ }^{1}$ Based on frequency of occurrence of participants with achievement scores within each of the Borderline, Low Average, Average, High Average, Superior and Very Superior categories (as defined within the WRAT3)
} 


\section{Table 1: Comparison of behavioural and EDA data}

\begin{tabular}{|c|c|c|c|}
\hline & ADHD (N=15) & Controls $(\mathrm{N}=15)$ & \\
\hline & Mean (S.D.) & Mean (S.D.) & $\mathrm{P}$ \\
\hline Errors of commission & $5.4(3.6)$ & $2.9(1.7)$ & 0.03 \\
\hline Errors of omission & $1.58(1.36)$ & $0.5(0.46)$ & 0.008 \\
\hline Mean GoRT & $682.4(92.5)$ & $685.9(112.1)$ & NS \\
\hline Mean GoRT variability ${ }^{2}$ & $0.24(0.08)$ & $0.16(0.06)$ & \\
\hline \multicolumn{4}{|l|}{0.007} \\
\hline Post-withhold SCR & $0.25(0.26)$ & $0.27(0.22)$ & NS \\
\hline Post-commission error SCR & $0.37(0.38)$ & $0.691(0.49)$ & 0.05 \\
\hline Post-cue SCR & 0.524 (0.39) & $0.639(0.43)$ & NS \\
\hline
\end{tabular}

Post-hoc tests revealed a significant increase in SCR to commission errors relative to correct withholds in the control group $[F(1,28)=18.67, \mathrm{p}=0.000]$ that was absent in the ADHD group $[F(1,28)=1.55, \mathrm{p}=0.223]$. These differences are illustrated in Figure 1 below. After controlling for the effects of group, age and IQ the post-error vs. post-withhold SCR difference accounted for a significant percentage of the variance in commission errors $\left[\underline{\mathrm{R}}^{2}\right.$ change $=0.10, F(1,25)=3.66, \mathrm{p}=0.035,1$-tailed $\left.{ }^{3}\right]$

\section{INSERT FIGURE 1 ABOUT HERE}

Analysis of reaction times for individual stimuli, as illustrated in Figure 2 indicated equivalent post-error slowing in ADHD and control groups. Comparing RT to the digits 2 and 4, a repeated-measures ANOVA revealed a significant main effect of Stimulus $[F(1,28)=16.38, \mathrm{p}=0.000]$, no Group by Stimulus interaction $[F(1,28)=0.072, \mathrm{p}=0.79]$ and no main effect of Group $[F(1,28)=0.591, \mathrm{p}=0.45]$ indicating that across groups there was

\footnotetext{
${ }^{2}$ GoRT variability was calculated as a coefficient of variability defined as Mean $\mathrm{SD}_{\mathrm{GoRT}} / \mathrm{Mean}_{\mathrm{GoRT}}$.

${ }^{3}$ Based on the a-priori assumption that a reduced arousal response to an error would increase the likelihood of future errors
} 
an over all slowing of RT after an error. A further repeated-measures ANOVA was conducted to verify that this post-target slowing was specific to errors. A main effect of Response (post-commission slowing vs. post-withhold slowing) was found $[F(1,28)=11.1$, $\mathrm{p}=0.002]$ with no Group by Response interaction $[F(1,28)=0.41, \mathrm{p}=0.53]$ or main effect of Group $[F(1,28)=0.61, \mathrm{p}=0.441]$. This indicates that both the control and ADHD group children slowed more after a commission error than they did after a successful withhold and suggests comparable awareness of errors across groups.

\section{INSERT FIGURE 2 ABOUT HERE}




\section{Discussion}

The present study confirms the existence of a sustained attention deficit in ADHD found in previous work [4-6] using a modified version of the SART. ADHD children made more errors of commission and omission and showed significantly more variability in their response times than control children. The present study extends this work with the finding that children with ADHD exhibited a reduced electrodermal response to errors that predicted sustained attention deficits.

The findings of this study indicate that the SCRs of ADHD children were comparable following an error of commission and a successful withhold, whereas the SCRs of the control group were significantly larger following a commission error, relative to a successful withhold. Given that we found no impairments in the SCRs of ADHD participants to successful withholds or to auditory cues our findings are similar to those of Zahn and Kruesi [8] indicating a selective impairment in error processing as opposed to a general EDA deficit. We propose two potential explanations for this finding.

The first explanation for the selective impairment in error processing in ADHD is that these children were less aware of making errors than the control group. However the clear increase in RT after an error, evident in Figure 2, was equally significant for both the ADHD and control groups indicative of comparable error-awareness. Nevertheless it should be noted that Shallice et al [6] reported reduced post-error slowing in a nonresponse locked version of the SART and so it remains possible that response locking within our paradigm minimised the potential for post-error slowing.

Assuming normal recognition of errors in the ADHD group, an alternative and perhaps more likely explanation is that the ADHD children had a reduced emotional response to errors. The SART imposes a special importance on the appearance of the no-go target and performance of the task is geared towards avoiding errors of commission. Hence we argue that errors should be the most emotionally significant event during SART performance. This is supported by the findings of increased SCR amplitudes to 
commission errors relative to successful withholds in the control group. The absence of any such increase in the ADHD group suggests that these two response types did not engender a differential emotional significance for the ADHD group. The findings of the present study mirror those of a study we have carried out with patients with traumatic brain injury (TBI) which also indicated attenuated SCRs to errors even after controlling for awareness by asking participants to say "hit” if they made an error of commission (O’Keefe, Dockree and Robertson, under review). In that study it was also found that in TBI, as in our ADHD sample, the SCR abnormalities were restricted to making errors.

According to Damasio’s [10] ‘somatic marker hypothesis’ our behaviour is monitored with respect to prevailing affective and motivational states. An important role of the somatic marker is to mark future outcomes as advantageous or disadvantageous and hence influence the selection of behaviour. Experiments with card-game gambling tasks find that the usual increase in autonomic arousal that is presumed to bias against risky behaviour is absent in ventromedial prefrontal patients [11]. Skin conductance response provides an index of this autonomic response. The data in the present study indicates an attenuation of error related autonomic arousal in ADHD that is consequential for sustained attention performance. Performance of a repetitive and predictable task like the SART is heavily dependent on the participant's ability to maintain an alert state. Equally however, if attention does wane, the participant's ability to respond to an error by mobilising attentional resources and returning to a more controlled response style is central to reducing the likelihood of future errors. The connection between electrodermal responsiveness to errors and overall error rates suggests that this EDA impairment contributes in some way to decreased post-error correction in the ADHD group. We propose that the absence of the normal physiological marker to indicate disadvantageous behaviour (for example an automatic or mindless response style) decreases the likelihood that the ADHD group children will alter their response style when an error is made. 


\section{Conclusion}

While further research with larger samples would be desirable, we argue that the increased rate of sustained attention failures in the ADHD group can be partially attributed to a reduced evaluation of error significance that in turn results in reduced post-error correction of response style. This position is supported in the present study by the finding that the relative difference between SCRs to errors of commission and SCRs to withholds was consequential for commission error rate. 


\section{References}

[1] American Psychiatric Association. Diagnostic and Statistical Manual of Mental Disorders (4th ed.). Washington DC: American Psychiatric Association, 1994.

[2] Castellanos, FX, Giedd, JN, Eckburg, P, Marsh, WL, Vaituzix, AC, Kaysen, D, Hamburger, SD \& Rapoport, JL Quantitative morphology of the caudate nucleus in attention deficit hyperactivity disorder. Am J Psychiatry 1994; 151(12): 1791-1796.

[3] Casey, B J, Castellanos, FX, Giedd, JN, Marsh, WL, Hamburger, SD, Schubert, AB, Vauss, YC, Vaituzis, AC, Dickstein, DP, Sarfatti, SE, and Rapoport, JL. Implication of right frontostriatal circuitry in response inhibition and ADHD. J Am Acad Child AdolesC Psychiatry 1997; 36(3): 374-383.

[4] Manly, T, Anderson, V, Nimmo-Smith, I, Turner, A, Watson, P, and Robertson, IH. The Differential Assessment of Children's Attention: The Test of Everday Attention for Children (TEA-CH), Normative Sample and ADHD Performance. Journal of Child Psycol. Psychiat. 2001; 42(8): 1065-1081.

[5] Micallef, S, Anderson, J, Anderson, V, Robertson, I, \& Manly, T. Sustained and selective attention in children with ADHD and Specific Learning Disabilities. Clinical Neuropsychological Assessment, 2, 1-23.

[6] Shallice, T., Marzocchi, G.M., Coser, S., Del Savio, M., Meuter, R. \& Ruminati, R.I. (2002). Executive function profile of children with ADHD. Dev Neuropsychol 2001; 21(1): 43-71.

[7] Shibagaki, M, Yamanaka, T, and Furuya, T. Attention state in electrodermal activity during auditory stimulation of children with attention-deficit hyperactivity disorder. Percept. Motor Skills 1993; 77: 331-338.

[8] Zahn, TP \& Kruesi, MJP. Autonomic activity in boys with disruptive behaviour disorders. Psychophysiology 1993; 30: 605-614.

[9] Mangina, C.A., Beuzeron-Mangina, J.H., \& Grizenko, N. Event-related brain potentials, bilateral electrodermal activity and Mangina-Test performance in learning disabled/ AHDD pre-adolescents with severe behavioural disorders as compared to agematched normal controls. Int J Psychophysiol 2000; 37:71-85

[10] Damasio, AR. Descartes' Error: Emotion, Reason and the Human Brain. New York: G.P. Putnam, 1994.

[11] Hinson, JM, Jameson, TL \& Whitney, P. Somatic markers, working memory and decision making. Cogn Affect Behav Neurosci. 2002; 2(4): 341-353

[12] Sturm, W, \& Willmes, K. On the Functional Neuroanatomy of Intrinsic and Phasic Alertness. NeuroImage 2001; 14: S76-S84. 
[13] Sergeant, J. The cognitive-energetic model: an empirical approach to AttentionDeficit Hyperactivity Disorder. Neurosci Biobehav Rev 2000; 24: 7-12.

[14] Sonuga-Barke, EJS. The dual pathway model of AD/HD: an elaboration of neurodevelopmental characteristics. Neurosci Biobehav Rev 2003; 27(7):593-604.

[15]Robertson, IH, Manly, T, Andrade, J, Baddeley, BT, and Yiend, J. 'Oops!':

Performance correlates of everyday attentional failures in traumatic brain injured and normal subjects. Neuropsychologia 1997; 35(6):747-758.

[16]Manly, T, Owen, AM, McAvenue, L, Datta, A, Lewis, GH, Scott, SK, Rorden, C, Pickard, J, \& Robertson, IH. Enhancing the sensitivity of a sustained attention task to frontal damage: convergent clinical and functional imaging evidence. Neurocase 2003; 9(4): 340-349.

[17]Tranel, D and Damasio., H. Neuroanatomical correlates of electrodermal skin conductance responses. Psychophysiology 1994; 31: 427-438.

[18]Zahn, TP, Grafman, J, and Tranel, D. Frontal lobe lesions and electrodermal activity: effects of significance. Neuropsychologia 1999; 37: 1227-1241.

[19]Kirley, A, Lowe, N, Hawi, Z, Mullins, C, Daly, G, Waldman, I, McCarron, M, O'Donnell, D, Fitzgerald, M, \& Gill, M. Association of the 480 bp DAT1 allele with methylphenidate response in a sample of Irish children with ADHD. Am J Med Genet 2003; $121 B(1): 50-54$.

[20]Angold, A, Predergast, M, Cox, A, Harrington, R, Simonoff, E, \& Rutter, M. The Child and Adolescent Psychiatric Assessment (CAPA). Psychol Med 1995; 25: 739-753.

[21]Holmes, J, Lawson, D, Langley, K, Fitzpatrick, H, Trumper, A, Pay, H, Harrington, R, \& Thapar, A. The Child Attention-Deficit Hyperactivity Disorder Teacher Telephone Interview (CHATTI): reliability and validity. Br J Psychiatry 2004; 184(1): 74-78.

[22]Conners, C. K. (1998). Rating scales in attention deficit hyperactivity disorder: use in assessment and treatment monitoring. J Clin Psychiatry 1998; 59(7): 24-30.

[23]Wilkinson, GS. The Wide Range Achievement Test (WRAT3). Wilmington Delaware, USA: Wide Range Inc, 1993.

[24]Conners, CK. Parent Symptom Questionnaire. Psycholopharmacology Bulletin 1985; 21: 816-822.

[25] Bellgrove, MA, Dockree, P, Aimola, L, \& Robertson, IH. Attenuation of spatial attention asymmetries with poor sustained attention. NeuroReport 2004;15(6):1065-1069. 


\section{Figure captions}

Figure 1. Mean Skin Conductance Responses for each Group to no-go targets as a function of No-go Response (Withhold vs. Commission Error)

Figure 2. Mean reaction times for each stimulus immediately before and after an error of commission (the digit 3 is the no-go target) 